\title{
Cavernomas supratentoriales múltiples y cirugía de la epilepsia: caso clínico
}

\author{
R. Rocamora*,***; P. Mendivil** y A. Schulze-Bonhage*
}

*Centro de Epilepsia, Universidad de Freiburg, Alemania; **Departamento de Neurocirugía. Universidad de Freiburg, Alemania. ***Hospital Ruber Internacional. Departamento de Neurología. Madrid. España.

\section{Resumen}

Introducción. La epilepsia asociada a múltiples lesiones cerebrales requiere una evaluación prequirúrgica detallada. Se describe el caso de una paciente con 9 malformaciones cavernomatosas asociadas a epilepsia refractaria al tratamiento médico. Se destaca la importancia de un detallado análisis neurorradiológico y electroencefalográfico.

Caso clínico. Se trata de una paciente diestra de $\mathbf{5 6}$ años con una historia previa de epilepsia refractaria con crisis parciales complejas y secundariamente generalizadas de 26 años de evolución. Un estudio no invasivo con video electroencefalografía demostró una zona epileptogénica temporal anterior izquierda. El estudio detallado de imagen de RM no reveló alteraciones en la formación hipocámpica del mismo lado. Se realizó una lesionectomía extendida de 2 cavernomas adyacentes. La paciente ha permanecido sin crisis durante 24 meses posteriores a la cirugía.

Conclusiones. Las crisis epilépticas fármaco-resistentes asociadas a cavernomas múltiples pueden ser diagnosticadas mediante estudios prequirúrgicos no invasivos y tratadas mediante procedimientos resectivos únicos.

PALABRAS CLAVE: Hemangiomas cavernosos. Cavernomas. Epilepsia. Cirugía.

Multiple supratentorial cavernomas and epilepsy surgery: case report

\section{Summary}

Introduccion. Epilepsy associated with multiple lesions requires a detailed preoperative evaluation. We report on a patient with 9 cavernous malformations associated with medically intractable seizures

Recibido: 15-09-08. Aceptado: 1-10-07 who became seizure free after a selective resection of two adjacent cavernous resection. The importance of a detailed neuroradiological and electroencephalographic analysis is stressed.

Case report. The patient was a right-handed 56-yearold woman with a 26-year history of complex partial and secondarily generalized seizures. A non-invasive video EEG monitoring revealed the epileptogenic zone was temporal anterior on the left. A detailed MRI-analysis showed an intact hippocampal formation. An extended lesionectomy of 2 adjacent cavernomas was done. The patient has been free of seizures for 24 months after surgery.

Conclusions. Medically intractable seizures associated with multiple cavernous malformations can be diagnosed by non-invasive preoperative monitoring and controlled by single resective procedures.

KEY WORDS: Cavernous hemangioma. Cavernoma. Epilepsy. Surgery

\section{Introducción}

Los cavernomas cerebrales son malformaciones vasculares relativamente habituales ${ }^{20}$ y constituyen una causa crecientemente reconocida de epilepsias parciales. Las manifestaciones clínicas más frecuentes son crisis epilépticas (38-51\%), hemorragias (11-32\%) y déficit neurológicos focales $(12-45 \%)^{8}$. De 296 casos analizados en una revisión de la literatura, 235 (79\%) sufrían de epilepsia aislada o asociada a otras manifestaciones ${ }^{12}$. Se ha señalado que aproximadamente un $4 \%$ de las epilepsias refractarias a medicamentos son causadas por cavernomas ${ }^{2}$.

Los cavernomas muestran una especial predisposición a provocar manifestaciones epilépticas. Si se los compara con otras lesiones cerebrales -como las malformaciones arterio-venosas y los tumores- con volúmenes y localizaciones casi idénticos, los cavernomas presentan el doble de probabilidad de provocar crisis epilépticas ${ }^{11}$. Estas crisis son también la mayor causa de morbilidad en este grupo de pacientes $^{4,19}$. Dentro de ellas se observan todos los tipos de 
crisis epilépticas, incluyendo formas simples, complejas y secundariamente generalizadas ${ }^{18,21}$. La fisiopatología de las crisis epilépticas está relacionada con la irritación neuronal ocasionada por el contacto con múltiples microhemorragias que contienen productos de degradación de la hemoglobina, así como la reacción gliótica asociada a ellas ${ }^{1,9}$.

La prevalencia de cavernomas en la población general no se conoce con certeza, pero estudios post mortem revelan una frecuencia entre 0.02 y $0.5^{5,13,16}$. Se ha estimado que la frecuencia de pacientes con cavernomas múltiples es de cerca del $23 \%$ del total ${ }^{12}$. Las lesiones múltiples ocurren mucho más comúnmente en casos hereditarios ${ }^{14}$. Estos están sobre-representados en la raza hispánica y en pacientes con historia familiar positiva. Se ha sugerido además que las lesiones múltiples son más comunes en mujeres ${ }^{15}$. Genes asociados han sido encontrados en familias hispanas, alemanas y francesas ${ }^{7,10,17}$.

En pacientes con cavernomas supratentoriales únicos en los que se demuestra una buena correlación entre la localización de la lesión y el origen de las crisis comiciales identificado mediante estudios neurofisiológicos, la indicación de resección quirúrgica es comúnmente aceptada. Sin embargo, pacientes con dos o más lesiones potencialmente epileptogénicas presentan, desde el punto de vista del diagnóstico pre-quirúrgico, un desafío importante. Efectivamente, no hay recomendaciones terapéuticas ni diagnósticas para el manejo de la epilepsia en estos pacientes, cuyas malformaciones vasculares presentan características evolutivas singulares. Es posible que muchos de ellos en la actualidad no sean remitidos para evaluación pre-quirúrgica debido a la reducida experiencia existente en el tratamiento de epilepsias focales con lesiones múltiples en general, y a un eventual escepticismo pronóstico en pacientes con cavernomas múltiples en particular.

Nosotros describimos aquí el caso de una paciente con múltiples cavernomas supratentoriales (en el marco de una cavernomatosis familiar) asociados a epilepsia fármacoresistente, que fue exitosamente tratada mediante un único procedimiento resectivo después de realizar un diagnóstico prequirúrgico no invasivo.

\section{Caso clínico}

Paciente diestra de 56 años de edad en el momento del diagnóstico pre-quirúrgico, que experimentaba crisis parciales complejas (CPC) y secundariamente generalizadas (CSG) desde los 30 años. Ya en los primeros años de enfermedad se comprobó el carácter resistente de la misma a pesar de las múltiples combinaciones de fármacos antiepilépticos (FAE) utilizados. Una información detallada de la semiología de las crisis no pudo obtenerse ni por parte de la paciente ni de los familiares en el momento de la admisión. La paciente sufría fundamentalmente CSG.
A través de una terapia con Levetiracetam (1500 mg/día) permaneció sin crisis entre los años 2001 y 2004. Posteriormente reaparecieron las CPC diurnas y nocturnas con una frecuencia de 1-2/ mes. Posteriormente a las crisis se observaba una amnesia del episodio y se describían posibles episodios psicóticos postictales recidivantes.

La paciente no presentó antecedentes patológicos durante el embarazo, parto o desarrollo posterior. En 1998 una ecografía abdominal reveló un conglomerado de estructuras císticas en el riñón izquierdo. En la historia familiar, se describe que la madre presentaba una epilepsia desde la juventud y que la hija de 37 años también sufría de crisis epilépticas asociadas a cavernomas cerebrales múltiples, aunque momentáneamente controlada con medicamentos.

Los FAE utilizados hasta el momento en diferentes combinaciones han sido: Carbamazepina, Oxcarbazepina, Valproato, Fenobarbital, Gabapentina, Fenitoína, Vigabatrina y Clobazam. En el momento de la admisión, la paciente estaba siendo tratada con una combinación de Pregabalina $300 \mathrm{mg}$ /día y Levetiracetam $1500 \mathrm{mg}$ /día. No se encontraron alteraciones en el examen neurológico de rutina.

\section{Diagnóstico pre-quirúrgico}

Imagen de RM: se describen múltiples cavernomas supra e infratentoriales, bi-hemisféricos (fig. 1). Entre los cavernomas de tamaño no puntiforme se encuentran dos temporo-mesiales izquierdos, uno caudal al hipocampo y otro en el giro para-hipocámpico; otro en la vecindad del hipocampo derecho (que muestra signos de sangrado antiguo en T2); otro en la cabeza del núcleo caudado izquierdo; cuatro en las regiones post-centrales bilaterales y al menos uno en el tronco cerebral. Las formaciones hipocampales bilaterales, el fornix y los cuerpos mamilares se encontraron radiológicamente intactas.

Video-EEG continuo: durante los 7 días de registro con electrodos esfenoidales, se observó un enlentecimiento rítmico theta-delta intermitente a nivel de la región temporal anterior izquierda y en forma independiente también temporal anterior derecha. La actividad interictal mostró sharpwaves (sw) en Sp1 y T3 (temporal anterior izquierdo) con una distribución $98 \%$ y $1 \%$ respectivamente. De forma aislada también se observaron sw en la zona temporal derecha con máximo en T4. Se registraron 7 crisis epilépticas (tanto diurnas como nocturnas), observándose una actividad rítmica theta temporal izquierda previa al comienzo de la primera manifestación clínica con propagación temporal derecha a partir de 6 segundos (fig. 2). En el análisis semiológico se describe un comienzo con taquicardia, automatismos orales, mirada fija y automatismos manuales izquierdos o bilaterales, seguidos de generalización secundaria con clonías de comienzo en el brazo derecho. En el 


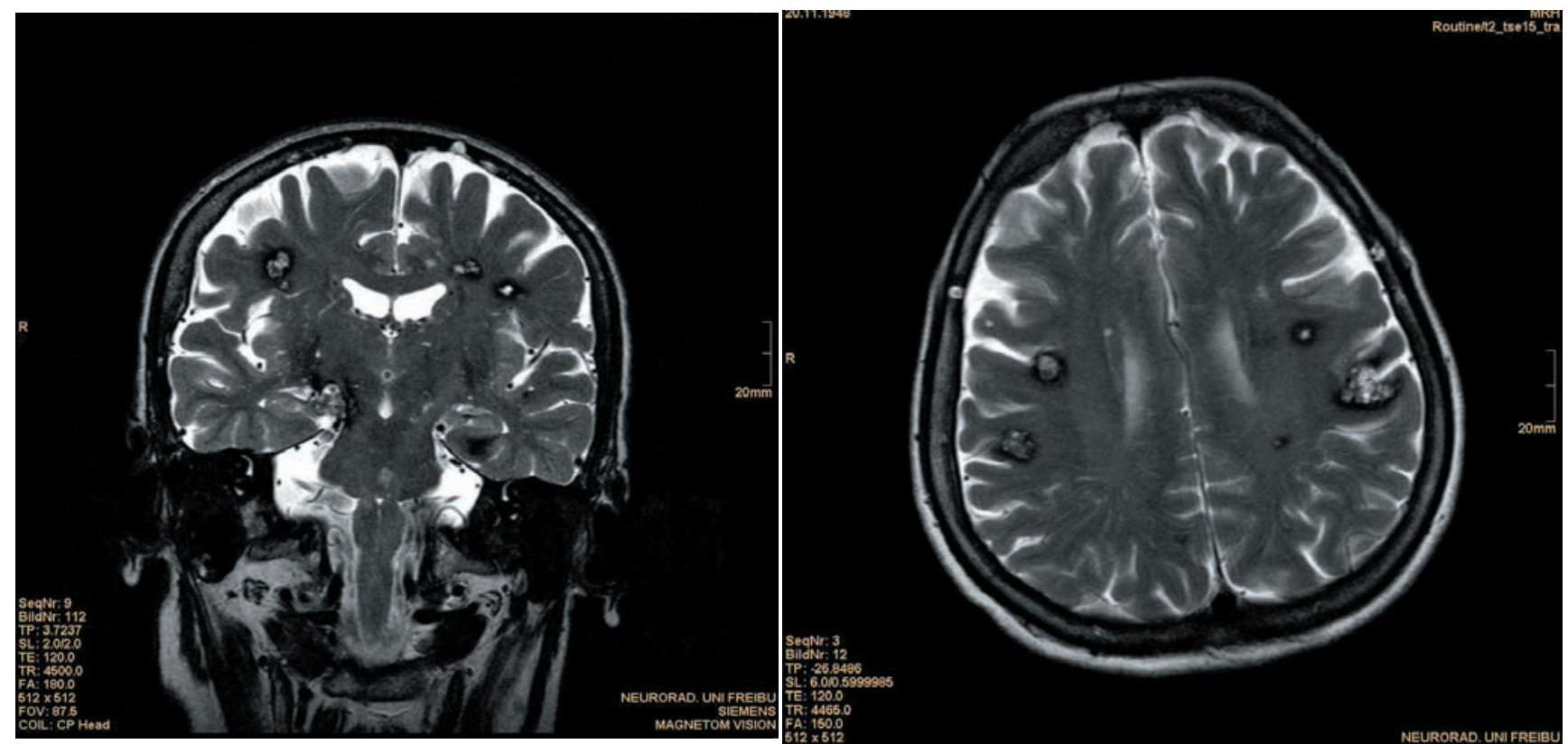

Fig. 1. En la imagen RM (T2) prequirúrgica se demuestran múltiples cavernomas supratentoriales bilaterales potencialmente epileptogénicos.
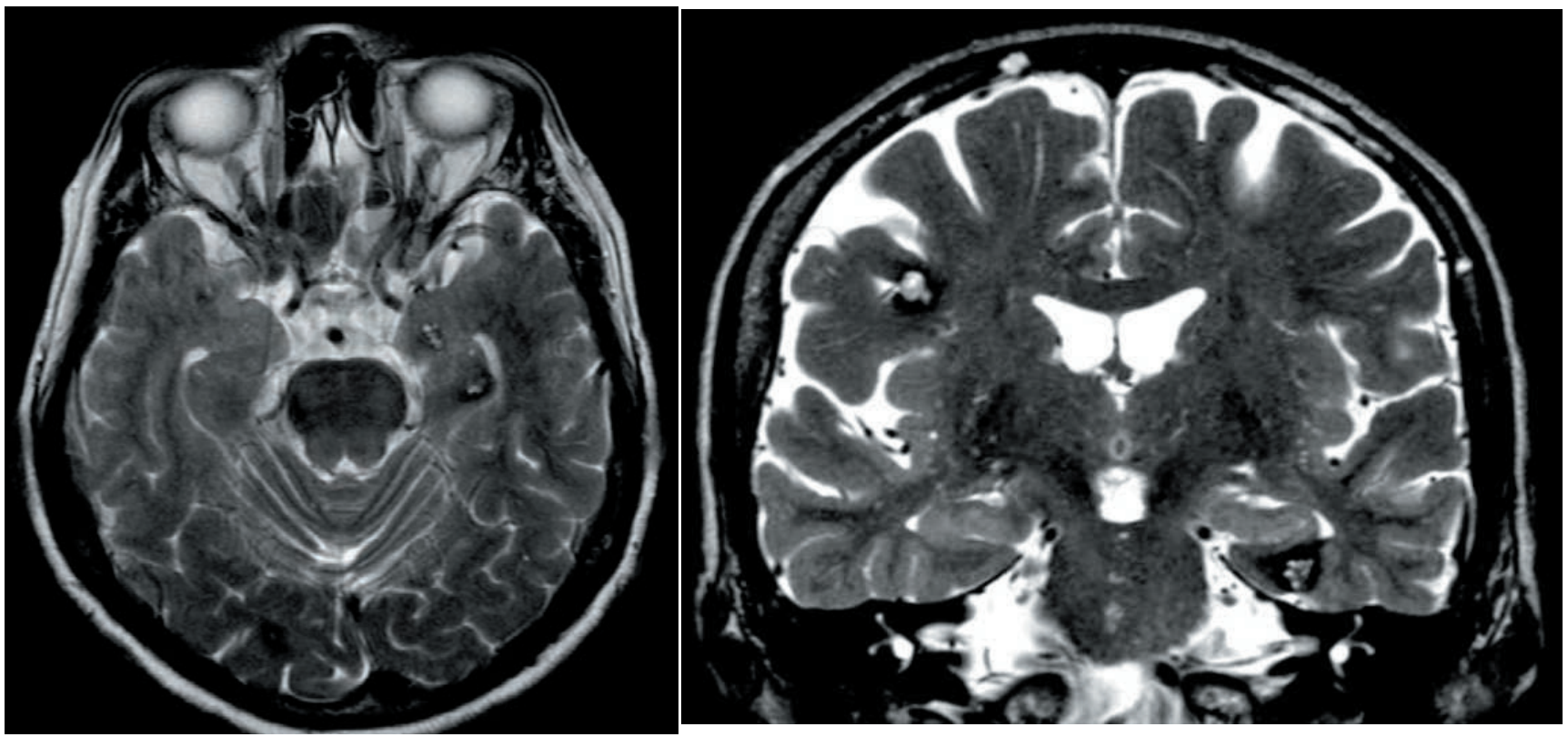

Fig. 2. Imagen de RM (T2) prequirúrgica: RM coronal y axial muestra los cavernomas epileptogénicos en el área temporal mesial izquierda.

período postictal se observó una desorientación de larga duración.

Estudio Neuropsicológico: disminución difusa de funciones corticales sin carácter localizador o lateralizante.

Examen psiquiátrico: psicosis postictales recidivante de días de duración y remisión espontánea.

Perimetría de Goldmann: examen perimétrico intacto.
Recomendación del estudio prequirúrgico: como resultado de la evaluación anterior se indicó la resección de los cavernomas temporomesiales izquierdos (fig. 3). Además, se recomendó respetar la estructura hipocámpica izquierda adyacente ya que un eventual sangrado espontáneo de los cavernomas temporales derechos hubiera significado para la paciente un riesgo elevado de sufrir un síndrome amnésico irreversible. 


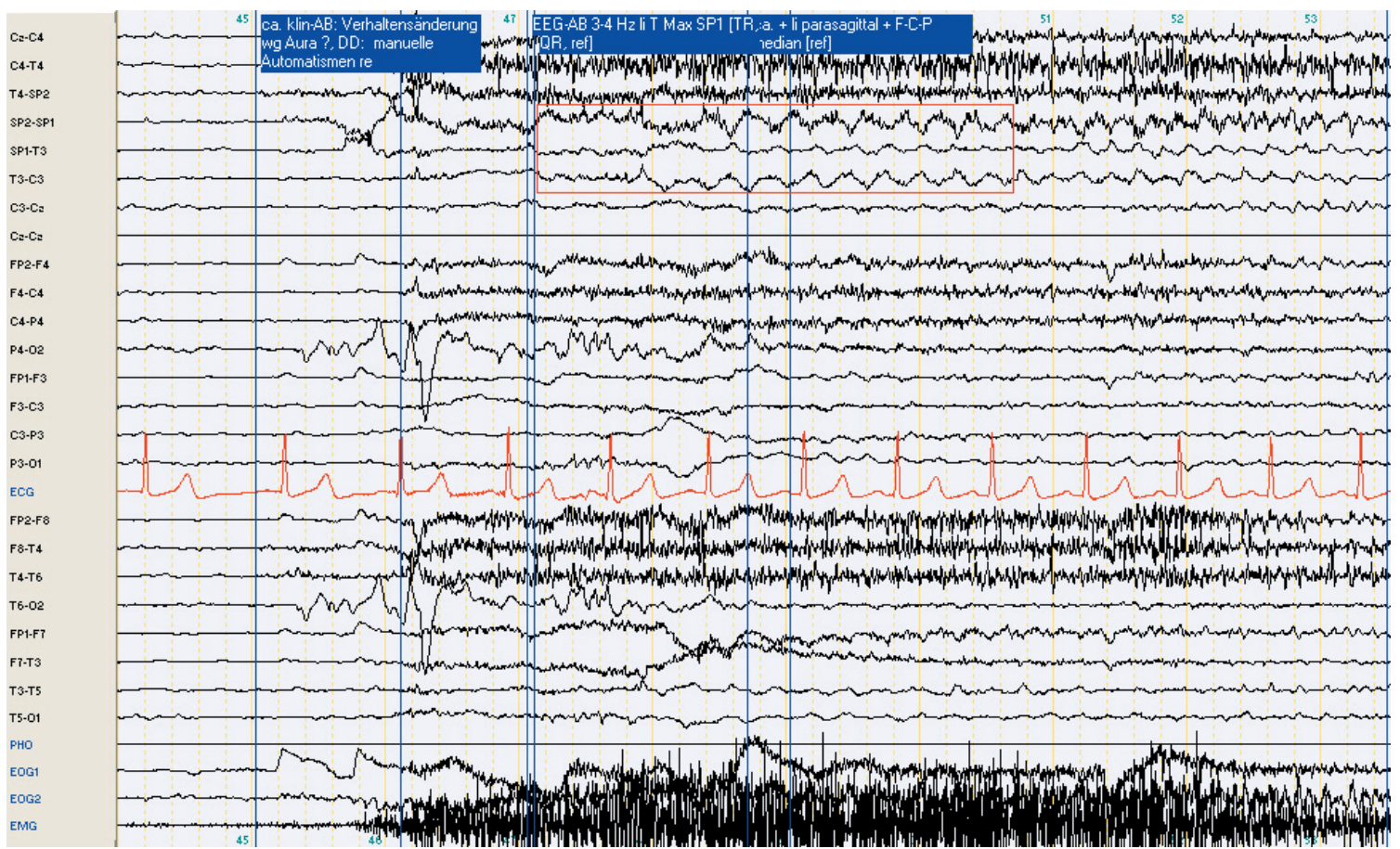

Fig. 3. Comienzo de actividad ictal electroencefalográfica 2 segundos posterior al comienzo de la primera manifestación clínica. Se observa una actividad ritmica theta con mayor amplitud temporal anterior izquierda (Sp1).

\section{Cirugía de la epilepsia}

A través de una trepanación fronto-lateral y bajo navegación ultrasónica se obtuvieron imágenes tridimensionales (3D) en las que se visualizaban los 2 cavernomas y las estructuras adyacentes. El anterior se localiza en el giro para-hipocámpico izquierdo y el posterior, inmediatamente caudal a la parte posterior del hipocampo del mismo lado.

Se procedió a la apertura del asta temporal izquierda a través de la cisura de Silvio y a la lesionectomía del primer cavernoma junto con la amígdala contigua. Posteriormente se procedió a la resección del segundo cavernoma localizado en la cara postero medial del hipocampo, respetando la formación hipocampal en su totalidad. En ambas resecciones se utiliza una técnica de lesionectomía extendida, extirpándose también el tejido cerebral gliótico y depósitos de hemosiderina que rodean al cavernoma y que pudieron visualizarse/palparse de forma directa por el neurocirujano durante el acto quirúrgico. Debido a que el primer cavernoma se encontraba en la parte basal de la amígdala adyacente, se procede a la resección de la misma ante la imposibilidad anatómica de su exclusión.

\section{Estudio anatomopatológico}

Se describen estructuras constituidas por dilataciones vasculares con tejido cerebral en los bordes. Se observan calcificaciones y fibrosis de los vasos con signos de hemo- rragias antiguas. Diagnóstico: angioma cavernoso.

\section{Control post-quirúrgico y pronóstico}

Durante los controles realizados a los 3, 6 y 24 meses posteriores a la cirugía, la paciente no experimentó crisis epilépticas (Engel Ia, Wiesser 1), permaneciendo con su tratamiento antiepiléptico.

\section{Discusión}

En el caso presentado describimos el tratamiento quirúrgico satisfactorio de una paciente con epilepsia resistente de 26 años de evolución que presentaba 8 cavernomas supratentoriales, todos ellos potencialmente epileptógenos. Esta constelación representa un caso particular de epilepsia focal en el marco de lesiones cerebrales múltiples ocasionadas por una enfermedad de origen hereditario. En el caso de la epilepsia intratable asociada a una lesión cavernomatosa única, si se demuestra una buena correlación entre los hallazgos de la actividad comicial electroencefalográfica, la semiología de las crisis y la localización del cavernoma, la lesionectomía extendida (resección del cavernoma más el área perilesional con depósitos de hemosiderina y gliosis reactiva) puede ser el tratamiento de elección ${ }^{4}$.

En pacientes con varias lesiones, se debe determinar si una o más de ellas están involucradas en la generación de las crisis epilépticas, aunque no debe descartarse a priori la 
posibilidad de que la epilepsia sea un fenómeno independiente de las lesiones observadas.

Desde el punto de vista radiológico debe determinarse qué lesiones demuestran señales compatibles con depósitos de hemosiderina por su reconocida mayor epileptogenicidad. Sin embargo, esta información debe ser considerada sólo como factor de riesgo relativo. El tamaño de la lesión, en esta paciente, tampoco pareció ser relevante. Los cavernomas parietales tenían un diámetro mayor que los temporales pero no demostraron carácter irritativo.

La localización probablemente tenga una mayor importancia. Si bien en este caso existían 8 diferentes lesiones, sólo aquellas localizadas en la cara mesial del lóbulo temporal izquierdo demostraron ser las generadoras de las crisis. No obstante, también se detectó actividad irritativa interictal en el lóbulo temporal derecho donde se encontraba otro cavernoma con signos de sangrado antiguo.

Por esto, es posible que la potencialidad de un cavernoma para generar crisis epilépticas dependa también del lóbulo comprometido y su singular cito-arquitectura. Otro elemento que no debe ser soslayado en el análisis radiológico es la evaluación detallada de las estructuras hipocámpicas. En este caso pudo respetarse el hipocampo adyacente y así evitar posibles secuelas neuropsicológicas. También debe considerarse especialmente la posibilidad del desarrollo de una patología dual en el transcurso del tiempo debido a un fenómeno de kindling. Es decir, la existencia de una esclerosis hipocampal asociada a otras lesiones temporales ${ }^{3}$. En general, los pacientes con cavernomas deben ser sometidos a un protocolo de Imagen de RM que incluya secuencias T1, T2, FLAIR y echo gradiente. El objetivo primordial del mismo es mostrar todas las lesiones existentes, el tamaño, la localización, señales de sangrado antiguas o recientes y por último descartar la presencia de patología dual.

Desde el punto de vista neurofisiológico, puede ser de ayuda la diferenciación del tipo de patrón electroencefalográfico observado al comienzo de la actividad ictal ${ }^{6}$. Se ha descrito que en la esclerosis hipocámpica el comienzo de crisis en el EEG se caracteriza por una actividad theta rítmica, mientras que en lesiones corticales, por una actividad de alta frecuencia y baja amplitud, LAFA (Low Amplitud Fast Activity). Sin embargo esta diferenciación sólo es útil cuando se logra detectar el comienzo de la crisis electroencefalográfica en una etapa temprana. No puede descartarse que en lesiones vasculares profundas se produzca primero una propagación hacia estructuras mesiales. En nuestra experiencia, se puede observar actividad theta-delta rítmica como comienzo de crisis en lesiones cavernomatosas que no se distinguen de las de origen hipocámpico. En el caso de patología dual confirmada y epilepsia tempo- ral, debe considerarse la necesidad de monitorización electroencefalográfica invasiva con electrodos subdurales si es que una resección de ambas estructuras supusiera un riesgo cognitivo elevado. La actividad interictal en este tipo de patología no posee un carácter definitivo debido a la presencia de múltiples lesiones. El rol pronóstico de la actividad irritativa en cavernomas remanentes no ha sido estudiada.

En este aspecto, el uso intraoperatorio de ECoG para comprobar la completa resección del foco irritativo es una opción a considerar en cirugía de la epilepsia, especialmente en casos de lesiones extensas como malformaciones arterio-venosas, tumores o displasias corticales. Sin embargo, debido a la delimitación de las lesiones cavernomatosas, su uso se hace innecesario en la mayor parte de los casos. Por otra parte, el uso de electrodos de foramen oval, preconizado por el grupo de Zürich, no ofrece, según nuestra experiencia y en concordancia con la escuela de Bonn, ventajas diagnósticas relevantes en comparación con la capacidad discriminativa de los electrodos esfenoidales. Al contrario, la posibilidad de complicaciones peri/post operatorias y la incomodidad de la misma para el paciente hacen de esta una técnica poco ventajosa desde el punto de vista del análisis riesgo-beneficio.

En el caso de que la lesión cavernomatosa se encuentre en un área elocuente, es decir, en aquélla en que se espera la localización de función motora y/o de lenguaje, la implantación de electrodos invasivos subdurales para la delimitación de las mismas, es ineludible.

Desde el punto de la técnica quirúrgica, si existe más de un cavernoma en el área epileptógena, debe realizarse la lesionectomía extendida de todas las lesiones si es que no hay áreas elocuentes comprometidas. Con EEG de superficie (no invasiva) se ha demostrado que la capacidad de discriminación espacial del área irritativa es de aproximadamente $6 \mathrm{~cm}^{2}$. Por lo tanto, no es posible determinar mediante esta técnica la epileptogenicidad individual de cavernomas adyacentes. En nuestro caso, se procedió a la resección de 2 cavernomas contiguos junto a la amígdala por motivos de técnica quirúrgica, aunque ésta se encontraba intacta en el diagnóstico neurorradiológico. Esta decisión fue favorecida por la falta de evidencia de un rol neuropsicológico significativo de esta estructura, a diferencia de la formación hipocámpica. Si bien no puede descartarse completamente un rol de la amígdala extirpada en la generación de las crisis epilépticas, la falta de alteración estructural y/o volumétrica del complejo amígadlohipocámpico junto a los signos de sangrado antiguo del cavernoma adyacente, hacen esta posibilidad muy poco probable.

En relación al pronóstico post quirúrgico en este grupo de pacientes, es posible que sea tan bueno como el de 

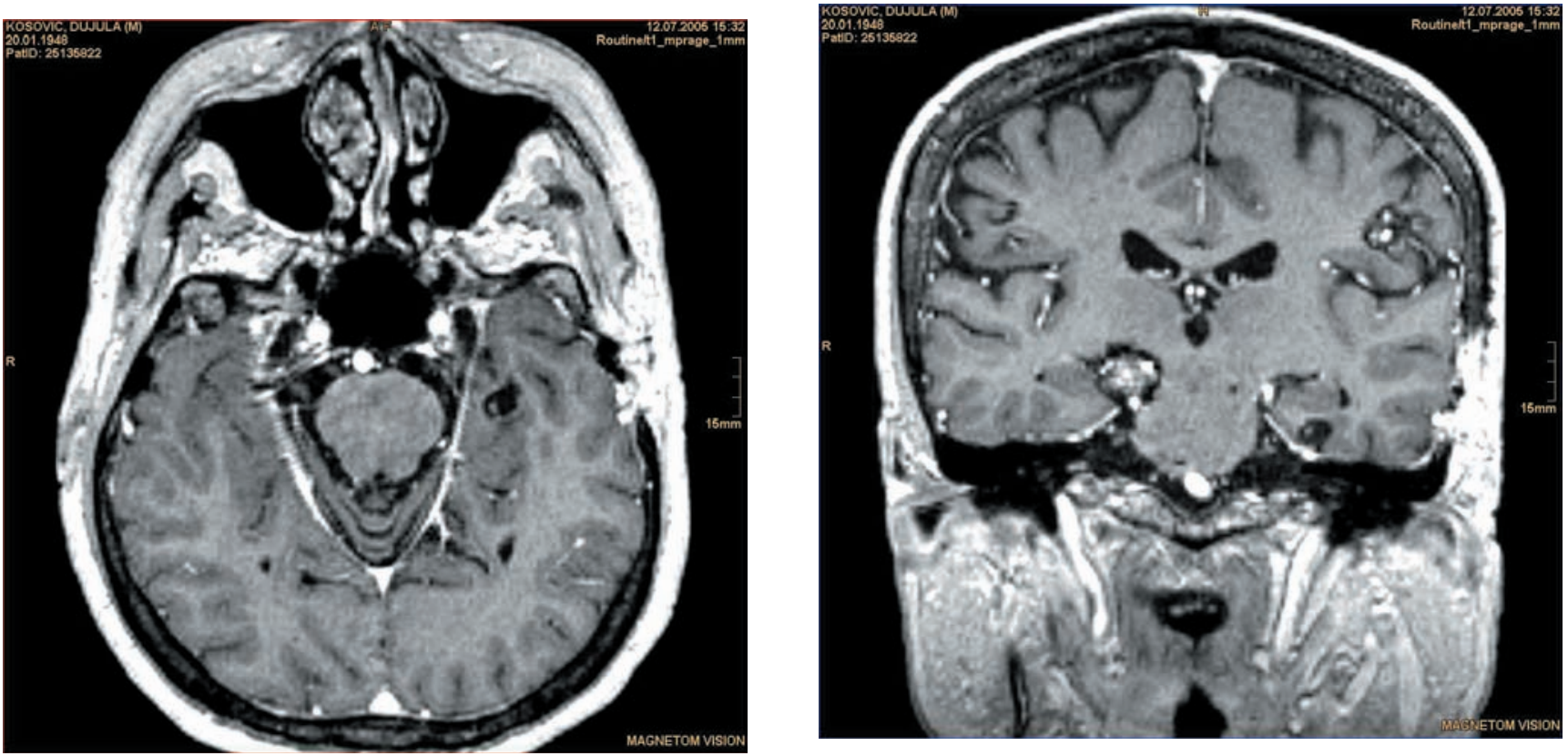

Fig. 4. Imagen de RM (T1 con Gadolinio) postquirúrgica: en el control de rutina, 3 meses posterior a la lesionectomía, se observa la zona de resección temporomesial izquierda.

cavernomas únicos. Nuestra paciente en particular ha permanecido libre de crisis hasta los 24 meses posteriores a la resección quirúrgica, demostrándose así la exactitud del procedimiento diagnóstico y quirúrgico (fig. 4).

La posibilidad de la generación de nuevos cavernomas (de novo) ha sido demostrada especialmente en casos con predisposición genética ${ }^{22}$. Esta debe ser considerada y discutida con los pacientes en el momento de evaluar una opción quirúrgica, así como la posibilidad de que otras lesiones tomen un carácter epileptogénico en el transcurso del tiempo. Al menos desde el punto de vista teórico, la probabilidad de permanecer libre de crisis sería algo menor en comparación con otras formas de cirugía de la epilepsia con lesiones más estables. Por otra parte, no debe olvidarse el hecho de la comorbilidad familiar y de que otros órganos puedan presentar también malformaciones cavernomatosas. Por esto, se recomienda la realización de una RM cerebral en familiares asintomáticos "en riego" una vez alcanzada la pubertad. En caso de sospecha de cavernomatosis familiar, el consejo genético debería llevarlo a cabo un especialista versado en el tema ${ }^{17}$.

Como conclusión, se demuestra que en el caso de cavernomas múltiples es posible detectar con fiabilidad la lesión responsable de la generación de crisis epilépticas y que ésta puede ser exitosamente tratada mediante cirugía. Un estudio invasivo no fue necesario y probablemente no lo sea en la mayoría de estos casos. Por lo tanto, un diagnóstico prequirúrgico debería considerarse siempre en etapas tempranas en todo paciente con cavernomas múltiples y epilepsia refractaria.

\section{Bibliografía}

1. Awad, I.A., Robinson, J.: Cavernous malformation and epilepsy, in Awad IA, Barrow DL, (eds): Cavernous Malformations. Park Ridge, AANS, 1993: 49-63.

2. Cappabianca, P., Alfieri, A., Maiuri, F., Mariniello, G., Cirillo, S., de Divitiis, E.: Supratentorial cavernous malformations and epilepsy: seizure outcome after lesionectomy on a series of 35 patients. Clin Neurol Neurosurg. 1997; 99: 179183.

3. Cascino, G.D., Jack, C.R. Jr, Parisi, J.E., et al.: Operative strategy in patients with MRI-identified dual pathology and temporal lobe epilepsy. Epilepsy Res 1993; 14: 175-182.

4. Cohen, D.S., Zubay, G.P., Goodman, R.R.: Seizure outcome after lesionectomy for cavernous malformations. J Neurosurg. 1995; 83: 237-242.

5. Del Curling, O. Jr, Kelly, D.L. Jr, Elster, A.D., Craven, T.E.: An analysis of the natural history of cavernous angiomas. J Neurosurg. 1991; 75: 702-708.

6. Ebner, A., Pannek, H.W., Schulz, R., Hoppe, M., Schmitt, J.J., Wörmann, F.G.: Erfolgreiche epilepsiechirurgische Behandlung eines Patienten mit fokaler Epilepsie und multiplen zerebralen Läsionen. Z Epileptol 2006; 19: 39-44.

7. Gûnel, M., Awad, I.A., Finberg, K., et al.: A founder mutation as a cause of cerebral cavernous malformation in Hispanic Americans. N Engl J Med 1996; 334: 946-951.

8. Hsu, F.P.K., Rigamonti, D., Huhn, S.L.: Epidemiology of cavernous malformations. En: Awad IA, Barow DL, eds. Cavernous malformations. Park Ridge: American Association of Neurological Surgeons Publications Committee, 1993: 13-23. 
9. Kraemer, D.L., Awad, I.A.: Vascular malformations and epilepsy. Epilepsia 1994; 35: 30-43.

10. Labauge, P., Laberge, S., Brunereau, L., et al.: Hereditary cerebral cavernous angiomas: clinical and genetic features in 57 French families. Lancet 1998; 352: 1892-1897.

11. Maraire, J., Awad, I.: Intracranial cavernous malformations: lesion behavior and management strategies. Neurosurgery 1995; 37: 591-605.

12. Moran, N.F., Fish, D.R., Kitchen, N., Shorvon, S., Kendall, B.E., Stevens, J.M.: Supratentorial cavernous haemangiomas and epilepsy: a review of the literature and case series. J Neurol Neurosurg Psychiatry. 1999; 66: 561-568.

13. Otten, P., Pizzolato, G.P., Rilliet, B., Berney, J.: 131 cases of cavernous angioma (cavernomas) of the CNS, discovered by retrospective analysis of 24,535 autopsies. Neurochirurgie. 1989; 35: 82-3, 128-131.

14. Perlemuter, G., Bejanin, H., Fritsch, J., Prat, F., Gaudric, M., Chaussade, S., Buffet, C.: Biliary obstruction caused by portal cavernoma: a study of 8 cases. J Hepatol. 1996 Jul; 25: 58-63.

15. Requena, I., Arias, M., López-Ibor, L., Pereiro, I., Barba, A., Alonso, A., Monton, E.: Cavernomas of the central nervous system: clinical and neuroimaging manifestations in 47 patients. J Neurol Neurosurg Psychiatry. 1991 Jul; 54: 590594.

16. Robinson, J.R., Awad, I.A., Little, J.R.: Natural history of the cavernous angioma. J Neurosurg. 1991; 75: 709-714.

17. Siegel, A.M., Bertalanffy, H., Dichgans, J.J., et al.:

\section{Comentario al trabajo Cavernomas supratentoriales múltiples y cirugía de la epilepsia: caso clínico de $\mathbf{R}$. Rocamora y cols.}

Los cavernomas cerebrales constituyen tal vez uno de los ejemplos paradigmáticos de la patología neuroquirúrgica, caracterizada por la extrema dificultad que supone realizar una aproximación pronóstica individual y una toma de decisiones diagnósticas y terapéuticas casi única para cada paciente, dada la complejísima combinación de variables que van a influir en la evolución de estas lesiones. Pero dentro de las múltiples posibilidades de presentación clínica y patológica de los angiomas cavernosos, la coexistencia de cavernomatosis cerebral múltiple y epilepsia farmacorresistente representa quizás el mayor de los desafíos para el neurocirujano, que debe enfrentarse al objetivo de la abolición de las crisis comiciales mediante una técnica quirúrgica única, que combine los requisitos de mínima invasividad junto con la necesidad de identificación del foco epileptógeno y su resección completa, muchas veces en la proximidad de áreas cerebrales elocuentes. ¿Qué grado de invasividad en la evaluación
Familial cavernous malformations of the central nervous system. A clinical and genetic study of 15 German families. Nervenarzt 2005; 76: 175-180.

18. Simard, J.M., García, B.F., Ballinger, W.J., Mickle, J.P., Quisling, R.G.: Cavernous angioma: A review of 126 collected ans 12 new clinical cases. Neurosurgery 1986; 18: 162-172.

19. Vaquero, J., Salazar, J., Martínez, R., Martínez, P., Bravo, G.: Cavernomas of the central nervous system: clinical syndromes, CT scan diagnosis, and prognosis after surgical treatment in 25 cases. Acta Neurochir (Wien). 1987; 85: 2933.

20. Voigt, K., Yasargil, M.G.: Cerebral cavernous haemangiomas or cavernomas: Incidence, pathology, Localization, diagnosis, clinical features and treatment. Neurochirurgia 1976; 19: 59-68.

21. Weber, M., Vespignani, H., Bracard, S., et al.: Intracerebral cavernous angioma. Rev Neurol 1989; 145: 429-436.

22. Wilson, C.B.: Cryptic vascular malformations. Clin Neurosurg. 1992; 38:,49-84.

Rocamora, R.; Mendivil, P.; Schulze-Bonhage, A.: Cavernomas supratentoriales múltiples y cirugía de la epilepsia: caso clínico. Neurocirugía 2008; 19: 257-266.

Correspondencia postal: Dr. Rodrigo Rocamora Zúñiga. Hospital Ruber Internacional. Departamento de Neurología. C/ La Masó 38. E-28034 Madrid. prequirúrgica debe exigirse para definir prequirúrgicamente el foco epileptógeno con la máxima precisión? ¿Qué estudios funcionales deben practicarse para preservar las áreas cerebrales elocuentes adyacentes al foco epileptógeno? Y finalmente, ¿qué tipo de abordaje y resección quirúrgica ha de realizarse para eliminar la sintomatología comicial? El trabajo de los doctores Rocamora, Mendivil y SchulzeBonhage, del Centro de Epilepsia y Departamento de Neurocirugía de la Universidad de Freiburg, nos proporciona muchas de las claves necesarias para abordar con éxito este desafío, mediante una exposición lógica y perfectamente argumentada de la toma de decisiones realizada en un paciente con cavernomatosis cerebral y epilepsia del lóbulo temporal. En este caso el tratamiento quirúrgico satisfactorio se basó en una aplicación individual de estudios diagnósticos progresivos, que se realizaron según el principio de mínima agresividad y máxima eficiencia en la información obtenida. Por ello todos los neurocirujanos 
tenemos mucho que aprender de casos individuales como el aquí descrito.

¿Sobre qué conocimientos fundamentales, tanto neurofisiológicos como de la neurociencia básica, debemos apoyarnos los neurocirujanos para abolir las crisis epilépticas de un paciente con cavernomatosis cerebral? Aunque el mecanismo exacto que induce las crisis comiciales farmacorresistentes asociadas a un cavernoma cerebral no se conoce todavía con precisión, sí se sabe que el principal causante son los depósitos de hemosiderina que microhemorragias de repetición (clínicamente silentes) producen en el tejido nervioso adyacente al cavernoma. Ya estudios clásicos experimentales de las décadas 60 y 70 demostraron en modelos de epileptogénesis animal que el hierro es un ión con propiedades muy epileptogénicas y éste es un constituyente principal de la hemosiderina ${ }^{4,16}$. Se ha observado que el hierro produce una inhibición de la recaptación del glutamato (neurotransmisor excitotóxico) liberado en las sinapsis, una función llevada a cabo principalmente por las células gliales, y puede que éste sea el mecanismo epileptogénico fundamental ${ }^{1}$. El hecho es que muy recientemente se ha comprobado que junto a la extirpación de la lesión cavernomatosa (lesionectomía), la resección quirúrgica del anillo de tejido nervioso gliótico infiltrado de hemosiderina que rodea a los cavernomas causantes de epilepsia farmacorresistente supone un pronóstico de abolición de crisis a largo plazo mucho más satisfactorio $^{2}$. Esta técnica quirúrgica, denominada lesionectomía extendida, consiguió incrementar el porcentaje de pacientes sin crisis a los 2 años de la intervención quirúrgica en un $35 \%$, lográndose la remisión de las crisis en un $59 \%$ de las lesionectomías extendidas frente a solo el $24 \%$ de los casos intervenidos mediante lesionectomía simple.

Bien, sabemos que una lesionectomía extendida puede permitirnos una eliminación de las crisis farmacorresistentes producidas por un cavernoma con mucha mayor efectividad, pero ¿existen otros factores reconocidos que influyan en el pronóstico postquirúrgico de las crisis epilépticas? Sí, y el más importante de todos es el tiempo de evolución de las crisis farmacorresistentes: cuanto mayor haya sido el tiempo en que el paciente haya sufrido crisis farmacorresistentes asociadas a lesión/es cavernomatosas cerebrales, menores son las posibilidades de abolición de las crisis con el tratamiento quirúrgico ${ }^{5,10}$. Este hecho apoya la hipótesis de Morrell, que establece la existencia de una epileptogénesis secundaria, mediada por las alteraciones causadas a distancia por la actividad epiléptica de un foco inicial o primario, que con el tiempo conducirían al desarrollo de focos epileptógenos secundarios e independientes a distancia del primero ${ }^{11}$. En consecuencia, la evaluación prequirúrgica y el tratamiento quirúrgico del paciente con crisis farmacorresistentes y cavernoma/s cerebrales debería realizarse de forma precoz.
En el caso de un paciente que presente una cavernomatosis cerebral múltiple con crisis parciales complejas farmacorresistentes, cuyo origen aparente sea uno de los lóbulos temporales según los estudios neurofisiológicos no invasivos (vídeo-EEG scalp) y en el que existan justo en este lóbulo una o varias lesiones cavernomatosas, bien a nivel cortical o adyacentes a estructuras mesiales (complejo amigdalo-hipocámpico), ¿qué tipo de estudio neurofisiológico y abordaje quirúrgico sería el más apropiado? Aquí nos hallamos ante el reto de determinar, previamente a cualquier intervención quirúrgica, si existe una lesión dual, es decir, si además de un foco epileptógeno situado en el área gliótica e infiltrada de hemosiderina que rodea al cavernoma, las crisis farmacorresistentes han inducido con el tiempo el desarrollo de otro foco epileptógeno secundario a nivel de las estructuras mesiales (amígdala, hipocampo, córtex entorrinal y córtex parahipocámpico), que impidan un pronóstico insatisfactorio de las crisis, aun realizando una lesionectomía extendida del cavernoma/s. Ante esta disyuntiva, de especial complejidad, debemos recurrir a una evaluación diagnóstica y a estrategias quirúrgicas propias de la cirugía de epilepsia, y el paciente requeriría, en circunstancias ideales, ser remitido a una unidad especializada en este tipo de cirugía, no por el hecho de que estas unidades impliquen una técnica quirúrgica más depurada o exquisita, sino porque aportan un estudio neurofisiológico, neuropsicológico y funcional cerebral mucho más exhaustivo y experto, y es este estudio el que debe guiar la resección quirúrgica. Por otro lado y ya desde una perspectiva de interés científico, (que desgraciadamente parece apartada de los objetivos asistenciales prioritarios de los servicios neuroquirúrgicos de nuestro país), los pacientes con afectación funcional del lóbulo temporal que puedan requerir estudios neurofisiológicos invasivos o intraoperatorios representan una fuente de conocimiento extremadamente valiosa sobre las relaciones entre estructura, función y manifestaciones psíquicas del cerebro, el campo de investigación más apasionante de la futura ciencia médica. Jamás debería perderse la oportunidad de aprender sobre el cerebro que proporcionan casos individuales neuroquirúrgicos, aunque ello suponga realizar exploraciones preoperatorios más exhaustivas, estudios intraoperatorios que alarguen el tiempo quirúrgico o recurrir a neurocientíficos de diversos campos que evaluen la importancia de los hallazgos histológicos, neurofisiológicos, clínicos o psicológicos del paciente, permitiendo así implicar realmente a la cirugía cerebral en la adquisición de conocimiento del cerebro humano.

La lesionectomía simple de los cavernomas cerebrales ha sido clásicamente el tratamiento quirúrgico estándar para lograr unos resultados aceptablemente satisfactorios del control de las crisis comiciales farmacorresistentes. Sin embargo, cuando se analizan los resultados de la 
lesionectomía en función de la localización del cavernoma, los resultados han sido desalentadores: menos del $25 \%$ de los pacientes con cavernomas localizados en el lóbulo temporal y extirpados mediante lesionectomía simple logran una remisión completa de las crisis ${ }^{3}$. Se ha comprobado que cuando las resecciones de lesiones tumorales del lóbulo temporal se realizan incluyendo una evaluación y en su caso resección del complejo epileptógeno asociado, guiada neurofisiológicamente mediante registros EEG, bien prequirúrgicos mediante electrodos invasivos o bien intraoperatorios con electrocorticografía, el control de las crisis a largo plazo es superior al $90 \%{ }^{8}$. No se ha podido dilucidar todavía si el fallo del control de las crisis tras lesionectomía simple de una lesión del lóbulo temporal se debe a una pérdida de neuronas inhibitorias a nivel del hipocampo como efecto secundario de las crisis epilépticas, manifestándose estas alteraciones clínicamente después de la intervención, o si la alteración sináptica es un evento ya responsable de las crisis farmacorresistentes, de forma independiente a la lesión, antes de la cirugía. Un estudio reciente sugiere que la hemosiderina depositada alrededor de las lesiones cavernomatosas induce una alteraciones de la actividad sináptica que conduciría a un daño neuronal permanente15. De lo que sí hay claras evidencias clínicas es que con la extirpación quirúrgica del hipocampo se logra un eficaz control de las crisis en muchas lesiones temporales, incluso cuando éstas no pueden ser extirpadas en su totalidad, debido a su localización posterior en áreas elocuentes ${ }^{6,14}$.

El principal problema asociado a la hipocampectomía quirúrgica es el hecho ampliamente reconocido de una pérdida permanente de los contenidos específicos de memoria que caracterizan la función mnésica hipocampal, ya sea una reducción de la capacidad mnésica verbal, en el caso de una hipocampectomía izquierda, o de capacidad mnésica figurativa en el de la hipocampectomía derecha ${ }^{9}$. Las secuelas neuropsicológicas derivadas de una hipocampectomía son siempre superiores en el caso de que no haya evidencias neurorradiológicas de esclerosis mesial (alteración patológica consistente en pérdida de la población neuronal $\mathrm{y}$ gliosis predominante en las regiones CA1 a CA3 del hipocampo $)^{7}$. La ausencia de esclerosis mesial en el caso aquí descrito y el elevado riesgo asociado de una pérdida de capacidad mnésica verbal en el paciente de haber practicado una hipocampectomía, recomendaban la preservación quirúrgica de esta estructura, tal y como se decidió tras comprobar la ausencia de déficits focales del lóbulo temporal en el estudio prequirúrgico neuropsicológico.

¿Con qué grado de invasividad deben estudiarse neurofisiológicamente las estructuras temporales mesiales para descartar la existencia de una actividad epileptiforme secundaria $\mathrm{o}$ independiente a una lesión o lesiones cavernomatosas? Este es un aspecto muy difícil de estan- darizar, en el que debe buscarse un equilibrio individual en el ratio riesgos versus beneficios de cada técnica, así como valorar el grado de experiencia y los resultados obtenidos con el empleo de cada tipo de estudio. En el caso aquí descrito el paciente fue estudiado con una técnica semiinvasiva, empleando electrodos esfenoidales para obtener un registro específico de las áreas mesiales, sin utilizar registro electrocorticográfico intraoperatorio. Con este estudio, en combinación con los datos neuropsicológicos y neurorradiológicos se ha obtenido un excelente pronóstico postquirúrgico, con remisión completa de las crisis comiciales sin realizar hipocampectomía, aunque hay que destacar que además de la lesionectomía extendida se extirpó el complejo amigdalino, lo que puede haber contribuido en gran medida a este resultado. Pero no puede generalizarse un pronóstico tan positivo en otros casos similares sin haber confirmado neurofisiológicamente la ausencia de epileptogenicidad hipocámpica con técnicas más discriminativas. Un caso muy similar al aquí presentado fue descrito por Adrian M. Siegel et al, de la Universidad de Zurich, y en este paciente las crisis epilépticas recurrieron tras la extirpación quirúrgica de un cavernoma temporal mesial adyacente al hipocampo izquierdo, a pesar de que los estudios neuropsicológicos y el test de Wada mostraron una indemnidad funcional de esta estructura ${ }^{12}$. Ello obligó a realizar una reintervención con registro electrocorticográfico intraoperatorio, incluyendo una tira de electrodos temporomesial, identificándose un foco epileptógeno temporal basal anterior, que fue extirpado, remitiendo así las crisis. Si hay dudas sobre la existencia de una lesión dual ante la presencia de lesiones extrahiopocámpicas con hallazgos electroencefalográficos no totalmente congruentes con la localización de la lesión, un estudio neurofisiológico invasivo, preoperatorio $\mathrm{y} / \mathrm{o}$ intraoperatorio, que incluya las estructuras temporales mesiales, puede ayudar a delimitar perfectamente el tipo de resección quirúrgica que debe realizarse $^{13}$.

\section{Bibliografía}

1 Awad, I.A., Rosenfeld, J., Ahl, J., Hahn, J.F., Luders, H.: Intractable epilepsy and structural lesions of the brain, Epilepsia 32: 179-186.

2. Baumann, C.R., Schuknecht, B., Lo Russo, G. et al.: Seizure outcome after resection of cavernous malformations is better when surrounding hemosiderin-stained brain also is removed Epilepsia 2006; 47: 563-566.

3. Cascino, G.D., Nelly, P.J., Sharbrough, F.W., Hulihan, J.F., Hirschorn, K.A., Trenerry, M.R.: Long-term follow-up of stereotactic lesionectomy in partial epilepsy: predictive factors and electroencephalographic results, Epilepsia 1992; 33: 639644.

4. Chusid, J.G., Kopeloff, L.M.: Epileptogenic effects of 
pure metals implanted in motor cortex of monkeys, J Appl Physiol 1962; 17: 697-700.

5. Ferroli, P., Casazza, M., Marras, C., Mendola, C., Franzini, A., Broggi, G.: Cerebral cavernomas and seizures: a retrospective study on 163 patients who underwent pure lesionectomy. Neurol Sci 2006; 26: 390-394.

6. Fish, D., Andermann, F., Olivier, A.: Complex partial seizures and small posterior temporal or extratemporal structural lesions. Surgical management. Neurology 1991; 41: 1781-1784.

7. Hermann, B.P., Wyler, A.R., Somes, G., Berry, A.D. $3^{\text {rd }}$, Dohan, F.C. Jr.: Pathological status of the mesial temporal lobe predicts memory outcome from left anterior temporal lobectomy. Neurosurgery 1992; 31: 652-657.

8. Jooma, R., Yeh, H.S., Privitera, M.D., Gartner, M.: Lesionectomy versus electrophysiologically guided resection for temporal lobe tumors manifesting with complex partial seizures. J Neurosurg 1995; 83: 231-236.

9. Katz, A., Awad, I.A., Kong, A.K. et al.: Extent of resection in temporal lobectomy for epilepsy. II. Memory changes and neurologic complications. Epilepsia 1989; 30: 763-771.

10. Moran, N.F., Fish, D.R., Kitchen, N., Shorvon, S., Kendall, B.E., Stevens, J.M.: Supratentorial cavernous hae- mangiomas and epilepsy: a review of the literature and case series. J Neurol Neurosurg Psychiatry 1999; 66: 561-568.

11. Morrell, F.: Secondary epileptogenesis in man. Arch Neurol 1985; 42: 318-335.

12. Siegel, A.M., Roberts, D.W., Harbaugh, R.E., Williamson, P.D.: Pure lesionectomy versus tailored epilepsy surgery in treatment of cavernous malformations presenting with epilepsy Neurosurg Rev 2000; 23: 80-83.

13. Sola, R.G., Hernando Requejo, V., Pastor, J. et al.: Epilepsia farmacorresistente del lóbulo temporal. Exploración con electrodos del foramen oval y resultados quirúrgicos. Rev Neurol 2005; 41: 4-16.

14. Sperling, M.R., Cahan, L.D., Brown, W.J.: Relief of seizures from a predominantly posterior temporal tumor with anterior temporal lobectomy. Epilepsia 1989; 30: 559-563.

15. Williamson, A., Patrylo, P.R., Lee, S., Spencer, D.D.: Physiology of human cortical neurons adjacent to cavernous malformations and tumors. Epilepsia 2003; 44: 1413-1419.

16. Willmore, L.J., Sypert, G.W., Munson, J.V., Hurd, R.W.: Chronic focal epileptiform discharge induced by injection of iron into rat and cat cortex. Science 1978; 200: 15011503.

J.M. Pascual

Madrid 